\title{
LA OBJECIÓN DE KRIPKE A LA TEORÍA DE LA IDENTIDAD MENTE-CUERPO
}

El objeto del presente trabajo es examinar las objeciones que presenta Saul Kripke ${ }^{1}$ a la teoría fisicalista que sostiene que los estados mentales son idénticos a ciertos estados (o procesos) físicos o corporales. Hay por lo menos dos versiones de la teoría de la identidad entre lo mental y lo físico que conviene distinguir aquí: a) la teoria que sostiene que la identidad se da entre tipos de estados mentales y tipos de estados físicos y $b$ ) la teorfa fisicalista que afirma que cada suceso mental fechable determinado es idéntico a un suceso físico particular también fechable, pero que niega la identidad entre tipos de estados mentales y tipos de estados físicos y, por tanto, la posibilidad de que existan leyes psicofísicas estrictas. Es la primera de estas dos teorías, la que pretende que las leyes psicofísicas son posibles, a la que Kripke opone con más fuerza sus objeciones.

Decir que un estado mental es idéntico a un estado ffsico equivale a aceptar que los términos mentales y físicos utilizados (por los fisicalistas) para enunciar la identidad psicofisica en cuestión, refieren en realidad a una y la misma cosa, estado o proceso. Así pues, afirmar, por ejemplo, que el dolor es idéntico a la estimulación de la fibra $C$, supone aceptar que las expresiones "dolor" y "estimulación de la fibra C" denotan una misma realidad. Dicho de otra manera, para que sea verdadero el enunciado "el dolor es idéntico a la estimulación de la fibra C", es necesario que ambos términos de la identidad tengan una misma referencia. Si un término mental $M$ y un término físico $F$ refieren a dos cosas o procesos distintos, el enunciado " $M=F$ " será falso, aun cuando $M$ y $F$ puedan estar de alguna manera correlacionados. La coocurrencia accidental de $M$ y $F$ no basta para establecer el enunciado de identidad correspondiente; como señala correctamente Kripke, afirmar que $\mathrm{M}=\mathrm{F}$ equivale a afirmar que $\mathrm{M}$ y $\mathrm{F}$ son necesariamente coocurrentes. (Kripke, 1972, p. 399.)

Las objeciones que Kripke opone a la teoría de la identidad entre lo mental y lo físico pueden examinarse críticamente desde dos perspectivas. La primera consiste en revisar el aparato conceptual que Kripke utiliza en sus artículos "Naming and Necessity" y "Identity and Necessity" con el fin de

1 Los trabajos de Kripke en donde aparecen las objeciones que consideraré son "Identity and Necessity" en M. Munitz (ed.) Identity and Individuation, New York Univ. Press, 1971 $y$ "Naming and Necessity" en Davidson y Harman (eds.) Semantics of Natural Language Dordrecht, Reidel 1972. En adelante estarán citados simplemente como 1971 y 1972 respectivamente. Hay traducción castellana de 1971 bajo el título Identidad y necesidad, Cuadernos de Crítica, no 7, UNAM, México, 1978. 
cuestionar la pertinencia o la validez de ciertas categorías y distinciones que introduce en esos trabajos y sobre las cuales basa sus objeciones; la segunda consiste en asumir provisionalmente el aparato conceptual de Kripke y argumentar con él en su propio terreno. Yo intentaré en este trabajo la segunda vía, esto es, no discutiré la validez de las tesis de Kripke sobre la naturaleza del nombrar, la designación rígida o los mundos posibles, ni pondré en cuestión la aceptabilidad de las modalidades de re o del esencialismo. Tampoco discutiré abiertamente la idea que Kripke tiene sobre la ciencia. Me limitaré a exponer, en esta primera parte, algunas ideas centrales de Kripke indispensables para entender sus objeciones a la teoría de la identidad y, en las secciones subsiguientes, intentaré analizar sus objeciones y mostrar que no son concluyentes, esto es, que dejan aún una puerta abierta por donde el fisicalista comprometido con la existencia de leyes psicofísicas y, por ende, con una teoría de la identidad entre tipos de estados mentales y tipos de estados físicos, puede aún escapar y sostener su posición como una alternativa conceptual real.

De acuerdo con Kripke, un enunciado de identidad tal como " $a=b$ ", es un enunciado acerca de los objetos designados por " $a$ " y por " $b$ " (esto es, no es un enunciado acerca de los nombres " $a$ " y " $b$ ", ni acerca de los posibles sentidos de " $a$ " y " $b$ ", ni de los posibles significados o conceptos asociados a estas expresiones) y lo que afirma es la existencia de una relación entre lo denotado por " $a$ " y lo denotado por " $b$ ". "La identidad" — dice Kripke"debe tomarse como la relación de una cosa consigo misma" (1972, p. 310). Al formular un enunciado de identidad, lo que asertamos es que un objeto es idéntico a sí mismo. Los enunciados de identidad no establecen sinonimias entre expresiones, ni pueden interpretarse como enunciados en el metalenguaje acerca de las expresiones " $a$ " y " $b$ ". Así, pues, el enunciado " $a=b$ " será verdadero si y sólo si " $a$ " y " $b$ " denotan al mismo individuo. Esto no implica que los enunciados de identidad sean triviales en el sentido de que no nos informen nada que no supiésemos de antemano y cuya verdad pudiese establecerse a priori. Puede constituir un verdadero descubrimiento empírico el hecho de que " $a$ " y " $b$ " denoten al mismo individuo.

Ahora bien, en "Identity and Necessity" (pp. 136 y ss.) Kripke ofrece un argumento formal para demostrar que si aceptamos: i) el principio de la indiscernibilidad de los idénticos de Leibniz, esto es, que $(x)(y)[(x=y) \rightarrow$ $(F x \leftrightarrow F y)]$; ii) la verdad de $(x) \square(x=x)$; iii) la existencia de propiedades modales tales como "ser necesariamente idéntico a $x$ " y $i v$ ) el principio de sustitutividad de los idénticos, tenemos que aceptar la consecuencia de que toda identidad verdadera es necesaria, esto es, que $(x)(y)[(x=y) \rightarrow \square(x=y)]$; dicho de otra manera, que no hay identidades contingentes. Decir que toda identidad verdadera es necesaria es equivalente a sostener que no sólo es verdadera en el mundo que de hecho se da, sino que sería verdadera en 
cualquier mundo posible o situación contrafáctica, esto es, que sería inconcebible una situación en la que el enunciado en el que se expresa fuese falso. (1971, p. 150). Para poder sostener consistentemente la tesis de que todas las identidades verdaderas son necesarias y la tesis de que las identidades pueden ser descubiertas empíricamente, Kripke tiene que introducir dos distinciones: 1) entre designadores rígidos y no rígidos, y 2) entre las nociones de necesidad y de aprioridad.

Un designador es una expresión del lenguaje cuya función semántica es referir a un individuo. Los nombres y las descripciones definidas son designadores. (1972, p. 254). ${ }^{2} \mathrm{Si}$ el designador designa al mismo individuo en todo mundo posible en donde el objeto existe, se dirá que es rígido; en tanto que sı designa a un individuo en el mundo que de hecho se da, pero pudiera designar a otros individuos en otros mundos posibles o situaciones contrafácticas, se dirá que es no rígido o accidental (1971, p. 145; 1972, pp. 269270). Así, por ejemplo, de acuerdo con Kripke, el nombre "Cicerón" es un designador rígido porque en éste y en cualquier otro mundo posible designaría a Cicerón, en tanto que la descripción "el autor de las Cartas a Ático" es no rígido, porque aunque de hecho designa a Cicerón en este mundo, podría haber designado a otro individuo en otro mundo posible. Cicerón no podría dejar de ser Cicerón, pero es algo perfectamente imaginable que Cicerón no hubiese escrito las Cartas a Atico y que alguien más hubiese escrito cartas cualitativamente idénticas a las que de hecho escribió Cicerón. Dicho de otra manera: es una situación concebible que llegásemos a descubrir que en realidad Cicerón no escribió las Cartas a Atico, sino que las escribió algún otro escritor romano, en tanto que no es concebible que descubriésemos que Cicerón no fue en realidad Cicerón.

Cabe notar que la distinción entre designadores rígidos y no rígidos no corresponde, de acuerdo con Kripke, a la distinción entre nombres y descripciones, ya que tanto los nombres (propios y de clases naturales) como ciertas descripciones, designan rígidamente a sus objetos. Serían descripciones rígidas, por ejemplo, aquellas que designaran a su referente mediante una propiedad esencial; por ejemplo, "la raíz cuadrada de 9" denota rígidamente al número 3, o "la energía molecular cinética media" denota rígidamente al calor. La admisión de designadores rígidos supone la aceptación de propiedades esenciales y, en este sentido, de las modalidades de re (1972, p. 264). ${ }^{3}$ Ahora bien, lo que Kripke sostiene es que si los términos que entran en un enun-

2 El referente de un nombre es la cosa nombrada; el de una descripción definida es la única cosa que satisface la descripción. Véase 1972, n. 3.

3 Las modalidades de re, por contraposición a las modalidades de dicto, conllevan la aceptación del esencialismo y la posibilidad de cuantificar en contextos modales (1971, p. 139). Aceptar las modalidades de dicto supone aceptar que hay entidades lingüisticas, como los enunciados, que son necesariamente verdaderas, pero no necesariamente supone la aceptación de hechos necesarios en la realidad no lingüistica. (Cfr. 1972, p. 264). 
ciado de identidad son ambos designadores rfgidos, y si el enunciado en cuestión es verdadero, entonces será necesario.4 Así, pues, dado que "Hesperus" y "Phosphorus" son designadores rigidos, y puesto que es verdadero que Hesperus es idéntico a Phosphorus, entonces será necesario el enunciado "Hesperus = Phosphorus". El hecho de que la verdad de esta identidad no haya sido establecida mediante razonamientos a priori, sino mediante la investigación empírica, no obsta para atribuirle necesidad a la identidad en cuestión.

Como vemos, es indispensable, también, trazar la distinción entre las nociones de necesidad y de aprioridad si se quiere defender la tesis de que las identidades verdaderas son necesarias. Tradicionalmente estas nociones, nos dice Kripke, habían sido confundidas o, por lo menos, usadas de manera intercambiable (1971, pp. 149-151; 1972, pp. 260-264). Muchos filosofos han sostenido que todos los conocimientos necesarios son obtenidos a priori $\mathrm{y}$, así mismo, que todo lo conocido a priori es necesario. Si efectivamente esto fuera el caso, esto es, si la necesidad y la aprioridad fuesen inseparables, sería insostenible la tesis de Kripke conforme a la cual toda identidad verdadera es necesaria, dado que la manera como llegamos a conocer un buen número de identidades es mediante la investigación empírica. Piénsese en las identidades "Hesperus $=$ Phosphorus" o "el calor $=$ la energía molecular cinética media". No bastaba reflexionar sobre "Hesperus" para descubrir que refiere al mismo astro al que refiere "Phosphorus" ni sobre el calor para percatarse de que es lo mismo que la energía molecular cinética media. Si hemos de sostener que estas identidades son necesarias, tenemos que deslindar la necesidad de la aprioridad.

Las categorías de necesidad y de aprioridad, afirma Kripke, pertenecen en realidad a dos campos distintos de la filosofía: La necesidad tiene su lugar en la metafísica, en tanto que la categoría de aprioridad lo tiene en la epistemología. (1971, p. 150; 1972, pp. 260-261). Un enunciado es necesario si y sólo si su falsedad es inconcebible, esto es, si no podemos señalar ninguna situación contrafáctica en la que el enunciado pudiese haber sido falso. Hablar de lo que podría o no haber sido el caso es asunto de la metafísica. Por otro lado, decir que un enunciado es a priori, es referirse al modo como conocemos (o podemos conocer) la verdad de ese enunciado. Un enunciado es a priori cuando no necesitamos apelar a la experiencia para establecer su verdad.

Una vez trazada la distinción anterior, Kripke muestra cómo, lejos de

4 Con respecto a las identidades expresadas en enunciados en los que alguno de los dos designadores utilizados es no rigido, no expresarán un hecho necesario. (Cfr. 1971, pp. 139 140 y 1972, p. 394). Siguiendo la teoria de las descripciones de Russell y aceptando su noción de "alcance de una descripción", podemos parafrasear dichas identidades de manera que lo único que afirman es la identidad de un objeto consigo mismo, 0 sea, que $x=x$, lo cual obviamente es necesario, y el hecho de que como cuestión de hecho empírica $F x$ y $G x$, en donde $F$ y $G$ son propiedades accidentales de $x$. 
haber una correspondencia biunívoca entre la clase de las verdades a priori y la de las verdades necesarias, son posibles todos los tipos de entrecruzamientos entre estas categorías y las de contingencia y aposterioridad. Si admitimos, como lo hace Kripke, las modalidades de re y aceptamos que las esencias pueden descubrirse empíricamente (1972, p. 312), esto es, que "la ciencia tiende a descubrir propiedades esenciales de los objetos que investiga" (1972, p. 330), tendremos que admitir la existencia de verdades necesarias establecidas a posteriori; las identidades teóricas en las que los términos de la identidad son dos designadores rígidos, por ejemplo, "el calor = la energía cinética media", serian el prototipo de esta clase de verdades. Hay también, de acuerdo con Kripke, verdades contingentes a priori (por ejemplo, "El metro patrón que está en el Museo de Pesas y Medidas en París en el tiempo $t$ mide un metro") asi como, desde luego, verdades contingentes a posteriori y necesarias a priori (estas últimas serían las verdades analíticas).

Dado todo lo anterior, podemos ya vislumbrar por dónde va a ir la primera parte del ataque de Kripke a la teoría de la identidad mente-cuerpo tal como ha solido presentarse históricamente. Tradicionalmente, los filósofos materialistas han aceptado, sin considerar que se trata de algo problemático, que las identidades psicofísicas, en el caso de ser establecidas, serían verdades contingentes. Esto es, han admitido que, aun cuando la verdad de la teoría fisicalista fuese establecida, seguiría siendo concebible que se diese un estado mental determinado sin que se diese su "correlato" físico. La explicación parcial de esto está en que probablemente no han distinguido las nociones de necesidad y de aprioridad. Piensan, con razón, que si la verdad de la teoría de la identidad ha de establecerse, esto no será sólo reflexionando sobre los conceptos que intervienen en las identidades psicofísicas, sino que será también el resultado de ciertos descubrimientos científicos, esto es, el producto de investigaciones empíricas; de esto (es decir, del carácter empírico de las identidades psicofísicas) pasan a sostener el carácter contingente de tales posibles identidades basados en la idea de que la experiencia no puede suministrar conocimiento de hechos necesarios. Kripke rechaza esta posición fisicalista después de mostrar que no hay tales identidades contingentes. La única salida que deja al filósofo materialista es aceptar la distinción entre necesidad y aprioridad y sostener que las identidades psicofísicas que supone verdaderas serían necesarias, si bien a posteriori. Pero en este caso, el defensor de la teoría de la identidad tiene que abandonar la idea de que sea concebible la existencia de un determinado estado mental sin su correlato físico o viceversa, esto es, tiene que deshacerse de la llamada "intuición cartesiana". El hecho de que tengamos esta intuición completaría la explicación de por qué consideran los materialistas que las identidades psicofísicas son contingentes. El defensor de la teorfa de la identidad tiene una tarea difícil: mostrar que lo que creíamos que era concebible en realidad no es concebible, que 
lo que creíamos que podíamos imaginar -esto es, que pudiera darse un cierto estado mental $M$ sin su correlato físico $F$-, en realidad no es algo que podamos imaginar. (1971, p. 163). La imaginabilidad de tales situaciones parece un hecho inapelable y está al fondo de la consideración de que las identidades psicofísicas son contingentes.

En suma, si el defensor de la teoría de la identidad sostiene que las identidades psicofísicas son contingentes, Kripke lo refuta mostrándole que no hay tal clase de identidades. Si pasa a sostener que son necesarias, tiene entonces que explicar la intuición cartesiana a manera de deshacerse de ella. Aquí es donde entraría la segunda parte de la objeción de Kripke, la cual es sin lugar a dudas la más fuerte. Considera que no es posible mostrar que la intuición cartesiana, esto es, la posibilidad prima facie de concebir que un estado mental $M$ puede existir independientemente de cualquier estado físico $F$ ( $y$, por tanto, que es concebible que $M \neq F$ ), sea un caso de mera ilusión de concebibilidad y que, por lo mismo, se trate de una intuición falsa, en tanto que si es posible mostrar que la aparente posibilidad de concebir la falsedad de las identidades teóricas de la ciencia no es más que una falsa apariencia y que, por tanto, en estos casos se trata de una mera ilusión de contingencia.

\section{La objeción de Kripke}

Kripke distingue tres formas distintas en las que se ha solido presentar la teoría de la identidad mente-cuerpo. (1972, p. 334):

1) La identidad de una persona con su cuerpo.

2) La identidad de dos sucesos singulares, esto es, un suceso mental irrepetible y particular (por ejemplo, esta sensación de dolor que estoy teniendo en este momento) con un suceso físico también irrepetible y particular (por ejemplo, mi tener en este momento determinada configuración neuronal en alguna región cerebral). Esta posición no admite que se puedan identiicar tipos de estados mentales con tipos de estados físicos, y por tanto niega la posibilidad de las leyes psicofísicas.

3) La identidad de un tipo de estado mental (por ejemplo, el dolor) con un tipo de estado físico (por ejemplo, la estimulación de las fibras $\mathrm{C}$ ). Esta versión del fisicalismo admite la posibilidad de leyes psicofísicas generales.

Cada una de estas modalidades distintas de la teoría materialista de la mente presenta dificultades peculiares, pero todas ellas parecen sujetas a un mismo tipo de objeción que ya Descartes adelantó y otros, incluyendo a Kripke, han insistido en presentar. ${ }^{5}$ La objeción cartesiana a la teoría de la

5 Colin McGinn ofrece un argumento para mostrar que la objeción de Kripke no se aplica a la versión 2 del fisicalismo. Véase su “Anomalous Monism and Kripke's Cartesian 
identidad enunciada en la forma (1), consiste en sostener que, dado que es concebible la existencia de la mente (o de experiencias) independientemente de la del cuerpo (o fenómenos neurofisiológicos), o, dicho de otra manera, puesto que es concebible que lo mental sea diferente de cualquier fenómeno corporal, entonces la mente (o los fenómenos mentales) y el cuerpo (o los fenómenos neurofisiológicos) no son idénticos, sino que son dos tipos de "sustancias" distintas. Algunos críticos de Descartes lo acusaron de cometer una falacia modal, esto es, de pasar injustificadamente de un enunciado de posibilidad a la afirmación categórica de que la mente (o la sustancia pensante) es distinta del cuerpo (o de la sustancia extensa). Este tipo de crítica, nos dice Kripke, es totalmente infundada (1971, p. 163, n. 19; 1972, pp. 334335) si consideramos que los términos "mente" y "cuerpo" usados en el argumento de Descartes son designadores rígidos. Esto es, si los términos con los que se enuncia la identidad designan rígidamente a sus objetos, no puede aceptarse simplemente la premisa cartesiana y negarse su conclusión. Si se acepta como verdadera la premisa acerca de la concebibilidad de los estados mentales sin sus respectivos correlatos físicos, o viceversa, tendremos que aceptar inevitablemente la conclusión dualista. (1972, p. 334). De manera que un defensor coherente de la teoría de la identidad, está obligado a mostrar que la aparente posibilidad de concebir que la mente no es idéntica al cuerpo, o que los estados mentales son distintos de los estados neurofisiológicos o físicos, no es más que una posibilidad ilusoria, es decir, que la intuición cartesiana es una intuición falsa. Hay quienes han sostenido que el argumento cartesiano no puede reformularse de manera que se aplique a la forma (2) de la teorfa de la identidad mente-cuerpo. En cambio, ha solido aceptarse que la objeción cartesiana se aplica a la forma (3) de la tesis materialista. En lo que sigue, la discusión se centrará en esta última forma debido a que es más clara y precisa que la 1), más fuerte que la (2), puesto que se compromete con la existencia de leyes psicofísicas, y es a la que Kripke opone con más fuerza sus argumentos.

Sean " $M$ " y " $F$ " designadores rígidos. Digamos que " $M$ " designa un tipo de estado mental determinado, digamos, el dolor, y " $F$ " un cierto tipo de estado f́́sico o neurofisiológico de las personas que tienen $M$. El defensor de la teoría de la identidad sostiene que $M=F$. Entonces, por el argumento de Kripke acerca de la necesidad de tales identidades, tendrá que sostener que $\square \mathrm{M}=\mathrm{F}$. Esto es, el materialista no podrá sostener consistentemente que $M=F$ y a la vez que es posible que $M \neq F$. Aceptar la posibilidad de que $\mathbf{M} \neq \mathbf{F}$ es equivalente a autorrefutarse.

$\mathrm{El}$ argumento $\mathrm{I}$ a continuación constituye la primera parte del ataque de Kripke en contra de la teoría de la identidad.

Intuitions", Andlisis 37, enero de 1977. Véase también: Olbeth Hansberg, "Monismo ạnómalo", Didnoia 1978, esp. pp. 160-162. 
Argumento I:

$$
\begin{aligned}
&\{1\}(1)(M=F) \rightarrow \square(M=F) \\
&\{2\}(2) \diamond \sim(M=F) \\
&\{\Lambda\}(3) \square(M=F) \rightarrow \sim \diamond \sim(M=F) \\
&\{2\}(4) \sim \square(M=F) \\
&\{1,2\}(5) \sim(M=F)
\end{aligned}
$$

Necesidad de la identidad. Concebibilidad de $(\mathrm{M} \neq \mathrm{F})$. Intuición cartesiana.

Por equiv. de op. mod. de 3 y 2 , por $M$. $T$. de 1 y 4 , por $M$. $T$.

$\mathrm{Si}$, como asumimos, " $\mathrm{M}$ " y " $\mathrm{F}$ " son designadores rigidos y aceptamos, dentro del espiritu de Kripke, el principio general que dice que cuando dos designadores rígidos denotan distintos individuos en algún mundo posible, entonces.denotarán necesariamente distintos individuos en todos los mundos en los que de hecho denoten algo, tendremos entonces que $(M \neq F) \rightarrow \square(M \neq F)$. Pensemos que si " $a$ " $\mathrm{y}$ " $b$ ". son designadores rígidos y designan dos individuos en el mundo actual, de manera que no es el caso que $a=b$, entonces en todo mundo posible en donde " $a$ " y " $b$ " denoten, designarán a dos individuos distintos, esto es, no será concebible ningún mundo posible en el que $a=b$. Si esto es asi, entonces tenemos que $a \neq b \rightarrow \sim \diamond a=b$. Pero el enunciado que afirma que $\sim \diamond \cdot a=b$, es equivalente al enunciado $\sim \sim \sim a=b$ y de este enunciado se sigue que $\square \sim a=b$. De manera que tenemos que aceptar que $a \neq b \rightarrow \square a \neq b$. Una vez munidos con este principio, a saber:

$$
\{6\}(6) \sim(M=F) \rightarrow \square \sim(M=F) \quad \begin{aligned}
& \text { Principio de la necesidad de la } \\
& \text { no identidad. }
\end{aligned}
$$

podemos tener la conclusión más fuerte:

$$
\{1,2,6\}(7) \square \sim(M=F) \quad \text { de } 5 \text { y } 6 \text {, por M.P. }
$$

Así, pues, a partir de la tesis de Kripke de la necesidad de la identidad y de la aceptación de la intuición cartesiana acerca de la concebibilidad de una situación en la que $\mathrm{M} \neq \mathrm{F}$, y añadiendo lo que he llamado "el principio de la necesidad de la no identidad", llegamos no sólo a la conclusión dualista, sino a la necesidad de dicha conclusión. Aquí el argumento comienza a parecer contraintuitivo, ya que resulta difícilmente aceptable que no sea ni siquiera concebible la falsedad de la tesis dualista.

Pensemos que el materialista puede construir el siguiente argumento, el cual diferirá del Argumento I sólo en que va a sostener en la premisa 2' que su posición es simplemente concebible, esto es, que es pensable que $M=F$. - Mediante este argumento el materialista llegará a la conclusión contraria a la que se llegó en el Argumento I. 
Argumento II:

$$
\begin{aligned}
\left\{1^{\prime}\right\}\left(1^{\prime}\right) \sim(M=F) \rightarrow \square \sim(M=F) \\
\left\{2^{\prime}\right\}\left(2^{\prime}\right) \diamond(M=F) \\
\left\{2^{\prime}\right\}\left(3^{\prime}\right) \sim \square \sim(M=F) \\
\left\{1^{\prime}, 2^{\prime}\right\}\left(4^{\prime}\right) \sim(M=F) \\
\left\{1^{\prime}, 2^{\prime}\right\}\left(5^{\prime}\right)(M=F) \\
\left\{1^{\prime}, 2^{\prime}\right\}(6) \square(M=F)
\end{aligned}
$$
Necesidad de la no identidad.
Concebibilidad de $M=F$. Intui- ción materialista.
de 2', por equiv. de op. mod.
de $\mathrm{l}^{\prime}$ y $3^{\prime}$, por $\mathrm{M}$. T.
de 4', por doble negación.
de 5 , por el principio de la ne- cesidad de la identidad.

En este Argumento II, partiendo de ciertas ideas del propio Kripke y de la "intuición materialista", conforme a la cual se acepta que al menos es pensable que $\mathrm{M}=\mathrm{F}$ (bajo peligro de reducción al absurdo de la posición materialista), se llega a la conclusión no sólo de que es verdadera la tesis de la identidad sino que es necesariamente verdadera. Si el argumento de Kripke en favor del dualismo obliga al materialista a mostrar que la intuición cartesiana es falsa, el argumento materialista obligaria al dualista a mostrar que la intuición materialista es falsa $o$, como diria Kripke, a mostrar el carácter ilusorio de esta intuición.

Cabe notar que en ambos argumentos basta partir de la concebilidad de una identidad (o no identidad) para establecer la correspondiente necesidad.

El problema obviamente parece estar en las premisas 2 y 2 '. Esto es, parece ser que en algún sentido del término "concebible", parecen efectivamente concebibles tanto $M \neq F$ como $M=F$. El hecho de que históricamente se hayan sostenido tanto posiciones dualistas como materialistas, nos obliga a aceptar, prima facie, que son pensables o concebibles tanto que $M \neq F$ como $M=F$. Al decir que son ambas situaciones "concebibles", ¿estamos simplemente aceptando ciertos hechos empíricos, esto es, el hecho de que históricamente ha habido filósofos y científicos que han sostenido la posibilidad de ambas situaciones? $\mathrm{O}$, por el contrario, cuando decimos que son ambas situaciones concebibles, zestamos aceptando algo acerca de lo que son las posibilidades reales, metafísicas? De esta cuestión nos ocuparemos en la siguiente sección.

Por el momento basta observar que, dado que ambas intuiciones, la cartesiana y la materialista, en el caso de ser tomadas conjuntamente conducén a una conclusión contradictoria, no pueden ser ambas verdaderas si se les toma como expresiones de posibilidades reales. Por lo tanto, en alguno de los dos casos se tratará de una mera ilusión de posibilidad.

Alguien podría decir que lo que hemos llamado la "intuición cartesana" y "la intuición materialista" en realidad no tienen el mismo status, esto es, que en tanto que la primera es una verdadera intuición con alcances metafisicos, la segunda no pasa de ser una mera hipótesis (falsa, por cierto) que han 
sostenido algunos filósofos y que sirve tal vez de guía en sus investigaciones a algún grupo de científicos. A esto podemos contestar (a) que no parece haber nada que las distinga formalmente, esto es, una afirma que es concebible la no identidad, la otra que es concebible la identidad; (b) decir simplemente que una tiene alcances metafísicos, en tanto que la otra no, es caer en una mera petición de principio; (c) que si la intuición materialista parece problemática o dudosa, tanto más dudosa resulta la intuición cartesiana, conforme a la cual un ser desprovisto de cerebro pudiera tener sensaciones. Los avances científicos no pueden menos que afectar nuestras intuiciones acerca de lo que es posible y de lo que no lo es. Dados los descubrimientos que se han efectuado en el campo de las neurociencias y de la psicofisiologia, resulta problemático, por contraintuitivo, aceptar la concebilidad de una situación en la que, por ejemplo, un maniquí sienta o piense o, como dice Kripke, la posibilidad (concebibilidad) de que alguien pueda tener experiencias y sin embargo descubramos que no tiene cerebro. ${ }^{\circ}$

Hemos visto que hay un sentido legitimo en el que parece que podemos decir que son concebibles ambas posiciones antagónicas; por otro lado tenemos que admitir que alguna de las dos intuiciones ha de ser falsa, so peligro de caer en una contradicción. Antes de revisar los argumentos de Kripke para defender que la intuición cartesiana es verdadera, veamos qué queremos decir cuando afirmamos que algo, una situación, es concebible.

\section{Concebibilidad y posibilidad}

Hay por lo menos dos maneras de interpretar la expresión "es concebible que $\mathbf{M} \neq \mathrm{F}^{\prime \prime}$ :

a) Una interpretación epistemológica, conforme a la cual diríamos que, dada la evidencia con la que contamos y los esquemas teóricos dentro de los que nos movemos, es posible que resulte ser el caso que $M \neq F$. Esto sería equivalente a sostener que, hasta donde sabemos, decir que $\mathrm{M}$ y $\mathrm{F}$ son distintos no implica contradicción. Nótese que la evidencia con la que contamos puede ser insuficiente o el marco teórico que utilizamos puede resultar equivocado. Parece ser que, en este sentido, distintas personas en un mismo tiempo $t$ o una misma persona en dos momentos distintos $t_{1}$ y $t_{2}$ pueden concebir posiciones contradictorias. (En un momento dado es concebible que la luz sean ondas, en otro es concebible que sean corpúsculos.) La evidencia con la que contamos en la actualidad parece hacer concebibles, en este sentido epistemológico, tanto que $M \neq F$ como que $M=F$. Esto es, estamos en una situación en la que nuestra ignorancia no nos permite descartar ninguna de las dos posibilidades.

b) Una interpretación metafísica, conforme a la cual lo concebible es lo 6 Cfr.j por ejemplo, 1971, p. 161 y 1972, p. 336. 
realmente posible, independientemente de que lo pensemos o no; lo que puede ser el caso en algún modo posible o situación contrafáctica independientemente de que lo conozcamos o lo imaginamos. ${ }^{7}$ En este sentido no serían posibles las situaciones que pudieran ser descritas mediante enunciados contra: dictorios. Si fuese el caso que $M=F$ (y si aceptamos el aparato de Kripke) efectivamente sería metafísicamente imposible que $\mathbf{M} \neq \mathbf{F}$, pero tal vez no fuese epistemológicamente inconcebible que $\mathbf{M} \leftarrow \mathbf{F}$ (por la sencilla razón de que no conocemos la verdad de $\mathrm{M}=\mathbf{F}$ ).

Usemos la expresión "concebibilidad" para réferirnos a lo epistemológicamente posible y hablemos de."posibilidad". para referirnos a lo metafísica o realmente posible.

Algo es concebible relativamente a un conjunto de conocimientos o creen: cias previas; si las creencias son falsas, lo que consideramos concebible en un momento $t$ puede no ser posible, esto es, podemos concebir situaciones imposibles .(por ejemplo, que la luz = ondas Fresnel). Puedo creer en estos momentos. que Saturno es un planeta cuya constitución química es $Q$ y en este sentido concebir que Saturno está hecho de $Q$. Pero si Saturno en realidad está hechó de otra sustancia distinta a $Q$ digamos de $Q^{\prime}$, es imposible que la constitución química de Saturno sea $Q$. Diriamos entonces que aunque es concebible (epistemológicamente posible) que Saturno esté hecho de $Q$ es metáfísicamente imposible que esté hecho de $Q$ puesto que está hecho en realidad de $Q^{\prime}$. Resúlta pues sostenible que lo que era- epistemológicamente posible (concebible) con respecto a un conjunto de creencias $C$ en un momento dado $t$, no sea concebible en un momento posterior $t^{\prime}$ en el que contamos con un conjunto $C^{\prime}$ de creencias. Antes de que los cientificos establezcan la verdad de una identidad del tipo $\dot{\alpha}=\beta$, es epistemológicamente posible tanto que $\alpha=\beta$ como que $\alpha \neq \beta$.

Ahora bien; cuando:la evidencia hace plausible sostener que es concebible tanto que $M \neq F$ como que $M=F$ (como parece ser el caso)-se estará hablando necesariamente de "concebibilidad" en el sentido de posibilidad epistemológica, pues, como argumentamos anteriormente, no puede tratarse de la posibilidad real o metafísica so pena de aceptar una contradicción. Pero si efectivamente se trata de que $M=F$ y $M \neq F$ son, hasta donde sabemos, epistemológicamente posibles, no podrá legítimamente pasarse de la concebibilidad de tales enunciados a la afirmación de la posibilidad real de las situaciones descritas mediante ellos. Esto es, dados los ejemplos antes mencionados, no contamos con una regla o principio general que garantice el paso de lo epistemológicamente posible a lo realmente posible.

Si esto es asi, entonces tenemos que el argumento cartesiano, o bien parte

7 Tal vez lo metafisicamente posible seria lo concebible sólo en el caso en que los esquemas teóricos o conceptuales que utilizásemos fuesen los correctos o adecuados $y$, por ende, contásemos con un conocimiento cabal de la realidad. 
de la concebibilidad (posibilidad epistemológica) de que $\mathbf{M} \neq \mathbf{F}$, en cuyo caso la premisa no es suficiente para establecer la conclusión deseada, esto es, que es metafísicamente posible que $M \neq F$, o bien introduce una premisa de indole metafísica con la única justificación de que su contraparte epistemológica ha sido aceptada, lo cual, como indiqué anteriormente, no es un paso generalmente garantizado. Si es metafísicamente posible que $M \neq F$, entonces será necesariamente falso que $\mathbf{M}=\mathbf{F}$. Para que el argumento cartesiano refute la posición materialista, es necesario que tome como premisa, no la posibilidad epistemológica de que $M \neq F$, sino la posibilidad metafísica; pero tomar esto último como premisa, sin más justificación que el hecho de que sea epistemológicamente posible que $M \neq F$, equivale a asumir lo que se quería probar. De la misma manera, si el argumento materialista partiese de la premisa que dice que es metafísicamente posible que $M=F$ para establecer la verdad de que $\square M=F$, se le podrian aplicar el mismo tipo de criticas.

En suma, el hecho de que sea concebible o imaginable que $M \neq F$, hace concebible (epistemológicamente posible) que sea metafísicamente posible que $\mathbf{M} \neq \mathbf{F}$, pero esto no basta para establecer la posibilidad real de $\mathbf{M} \neq \mathbf{F} .^{8}$

Como hemos señalado, las situaciones que concebimos las concebimos siempre dentro del marco de un conjunto de creencias. No podemos hablar de que $p$ sea concebible considerada como un átomo aislado, sin relaciones con otras creencias más o menos generales. Para poder mostrar que lo que epistemológicamente era concebible, digamos $p$, no es en realidad posible, es necesario mostrar que alguna de las creencias en relación a la cual resultaba concebible $p$, es una creencia falsa. Asi, por ejemplo, puedo mostrar que no es metafísicamente posible que Saturno esté hecho de $Q$ porque mi creencia de que $E$ era una prueba en favor, o evidencia suficiente, para establecer la verdad del enunciado en cuestión, era una creencia falsa, dado que en realidad lo que $E$ establece es la verdad de que Saturno está hecho de $Q^{\prime}$. Esto es posible hacerlo a posteriori. De la misma manera, para deshacer la intuición cartesiana o la materialista, es necesario mostrar que alguna de las creencias en las que estaban fundadas dichas intuiciones es en realidad falsa, y esto, también, parece ser una cosa que no podría llevarse a cabo más que si el desarrollo futuro de las neurociencias lo llegan a establecer.

La posición que sostengo es que tanto el argumento cartesiano como el materialista no pueden dar como justificación de sus premisas 2 y 2', respectivamente, el hecho de que $M \neq F$ o $M=F$ sean epistemológicamente posibles, porque, como hemos visto, esto en general no constituye una garantía de la

8 D. Wiggins, en "Identity, Designation, Essentialism and Physicalism", Philosophia, vol. 5, núm. 1-2, 1975, sostiene algo semejante: "La afirmación sincera de que uno ha imaginado [algo] diffícilmente puede demostrar la concebibilidad real [de ese algo]. Tal vez el que un hombre imagine que se da $p$ muestra al menos que es concebible que sea concebible que $p$. No lo sé." (p. 22). 
posibilidad metafísica. En este sentido, podriamos hablar de que hay en ambos argumentos, no una falacia modal, sino un problema de justificación. Si introducen 2 y 2', por otro lado, como afirmaciones de posibilidades metaffsicas sin más justificación, considero que están asumiendo justamente lo que se quería probar.

El problema al que nos enfrentamos aquí es de indole general: ¿Cómo es posible pasar de una posibilidad epistemológica a una posibilidad (o necesidad) real? ¿Es justificable pasar de la concebibilidad de una identidad a la necesidad de la misma?

Kripke distingue claramente el campo de la epistemología del campo de la metafísica y enfatiza en repetidas ocasiones la ilegitimidad de pasar, sin más argumentación, de una cuestión de indole epistemológica (por ejemplo, del carácter a priori de una verdad) a otra de índole metafísico (por ejemplo, al carácter necesario de la misma). Si considera que el argumento cartesiano puede reformularse de manera tal que no se le pueda acusar de dar este tipo de salto, es porque puede ofrecer otros argumentos (no la simple concebibilidad de que $\mathbf{M} \neq \mathbf{F}$ ) que le permiten defender el punto de vista conforme al cual la intuición cartesiana es la expresión de una genuina intuición metaf́́sica, y no de una mera posibilidad epistemológica, por contraposición a otro tipo de casos en donde también la falsedad de una identidad parece posible pero en donde puede mostrarse que dicha posibilidad es puramente ilusoria.

A continuación examinaremos el argumento de Kripke en favor de considerar que la intuición cartesiana expresa una auténtica posibilidad metafísica.

\section{La ilusión de contingencia en el caso de las identidades tedricas}

Las identidades teóricas tales como "el calor = la energía cinética media" o "la luz = un haz de fotones" contienen designadores rigidos y, por esta razón, si son verdaderas, son necesariamente verdaderas. (1971, p. 160, 1972, p. 331). Si esto es así, resultaría inconcebible la falsedad de una identidad teórica verdadera. Sin embargo, podemos tener frente a ellas la misma impresión de contingencia que el cartesiano tiene frente a una identidad psicofísica expresada mediante " $M=F$ " (por ejemplo "el dolor es idéntico a la estimulación de las fibras C"). Kripke explica por qué la impresión de contingencia en el caso de las identidades teóricas (por ejemplo "el calor = energía molecular cinética media") no es más que una mera ilusión de contingencia y pretende mostrar, a la vez, cómo no es posible aplicar el mismo tipo de explicación para eliminar la aparente contingencia en el caso de las identidades psicofísicas. Si no podemos disipar esa apariencia de contingencia, se tratará entonces de una genuina contingencia y la intuición cartesiana tendrá alcances metafísicos.

Para mostrar que la aparente contingencia de las identidades teóricas es 
ilusoria, Kripke introduce la noción de "descripción que fija una referencia". Una descripción puede ser usada para fijar la referencia de un designador, esto es, para identificar o seleccionar el objeto denotado por el designador. Así pues, usamos, por ejemplo, la descripción "lo que nos produce la sensación de caliente" para fijar la designación del término "calor", o "lo que nos produce la sensación de luminosidad" para fijar la referencia de la palabra "luz". Ahora bien, aunque "calor" es un designador rígido, la descripción que usamos para fijar su referencia es no rígida; esto es, seleccionamos' al calor mediante una propiedad accidental del calor, pues resulta concebible que. ese fenómeno externo que es el calor pudiera no producirnos la sensación de caliente. Si esto es así, nos dice Kripke, es efectivamente contingente que aquello que nos causa la sensación de caliente sea el calor y, por ende, la identidad "el calor = lo que causa en los humanos la sensación de caliente" no es necesaria, dado que "lo que causa en los humanos la sensación de caliente" no es un designador rígido. Pero esto de ninguna manera significa que sea también contingente que el calor = la energía cinética media. En este último caso tenemos una identidad necesaria, dado que es verdadera y contiene designadores rígidos. La ilusión de contingencia proviene, pues, del hecho de que seleccionamos el calor mediante una propiedad accidental (ser sentido de tal o cual manera). De esta manera, podemos éxplicar en términos generales la apariencia de contingencia de una identidad que utiliza los designadores rigidos $\boldsymbol{R}_{1}$ y $R_{2}$, señalando que las descripciones $D_{1}$ y $D_{2}$, mediante las cuales fijamos las referencias de $R_{1}$ y $R_{2}$, son descripciones accidentales. Si bien " $R_{1}=R_{2}$ " es necesario, no lo serán ni " $R_{1}=D_{2}$ " ni " $R_{2}=D_{1}$ " (ni desde luego tampoco " $D_{1}=D_{2}$ ), aún cuando estos últimos puedan ser verdaderos. Lo que nos da esa falsa impresión de contingencia con respecto al enunciado " $R_{1}=R_{2}$ " es que solemos fijar la referencia de $R_{1}$ mediante una descripción no rígida $D_{1}$ y asumir que " $R_{1}=R_{2}$ " es el mismo enunciadó que " $D_{1}=R_{2}$ "; lo cual; como hemos visto, no eś el caso. (1971, p. 160; 1972, pp. 333-4).

Pero ¿qué pasa en el caso de las identidades psicofísicas? Se trata, en.opinión de Kripke, también de identidades con designadores rígidos; "dolor" denota rígidamente la experiencia de dolor $y$, de acuerdo con Kripke, "estimulación de las fibras .C" denota rigidamente una determinada configuración neuronal. Sin embärgo, argumenta Kripke, puesto que ambos objetos designados son seleccionados mediante propiedades esenciales, no podemos alegar aquí que la apariencia de contingencia, como en el caso de las identidades teóricas, se deba a que hayamos fijado la referencia mediante propiedades accidentales. Seleccionamos el dolor porque lo sentimos, y es esencial al dolor ser sentido, y seleccionamos el estado cerebral por ser una configuración neuronal de tal y cual tipo, y es también esencial que sea dicha configuración neuronal. (1971, pp. 161-162; 1972, pp. 335, 340).

De esta manera, piensa Kripke, no sólo queda establecido que las identi- 
dades teóricas son realmente necesarias (a pesar de:que tenemos la falsa impresión de que es concebible su falsedad) sino que también ha mostrado que la intuición cartesiana resiste el tipo de explicación que se ofrece para disipar la apariencia de contingencia de las identidades teóricas, y permanece, por tanto, como una auténtica expresión de una posibilidad metafísica.

\section{'El caráçter no concluyente de la objeción de Kripke}

La argumentación de Kripke, sin embargo, no parece ser concluyente.

1) En primer lugar cabe señalar, que si bien es claro que seleccionamos el dolor por el hecho de que lo sentimos, lo cual efectivamente constituye una propiedad esencial del dolor (esto es, para que exista un dolor es necesario que se sienta), ${ }^{\ominus}$ no resulta: claro sostener que seleccionamos al estado cerebral por "ser dicho estado cerebral". Esto es, con respecto al dolor, es clato que la "evidencia": que nos justifica (si es que puede decirse tal cosa) para poder afirmar con verdad que tenemos un dolor, es el hecho de que lo sentimos; de manera que al fijar la referencia del término "dolor" mediante la descripción, por ejemplo, "esa sensación corporal que sentimos desagradable", efectivamente estamos usando una propiedad esencial del dolor para identifi: carlo. La "evidencia sensorial" es en este caso definitiva; hay una relación necesaria entre la evidencia y el objeto seleccionado y, en este sentido, podemos decir que seleccionamos el dolor mediante una propiedad esencial. En el caso del estado cerebral $\mathrm{F}$ (estimulación de la fibra $\mathrm{C}$ ), en cambio, no lo seleccionamos o identificamos por ser ese estado cerebral $F$, sino porque consideramos que, dentro del marco de una teoría, está correlacionádo con una determinada evidencia $E$ (la cual obviamente no incluye que sea sentido de ninguna manera). Así pues, podrfamos usar, por ejemplo, la descripción "el estado cerebral que se da cuando el encefalograma describe una gráfica del tipo G" o "el estado que se presenta cuando se registra mediante aparatos de laboratorio que hay liberación de una cantidad $n$ de acetil-colina en determinadas sinapsis", para fijar la referencia de la expresión "estimulación de las Fibras C". Ahora bien, la evidencia que nos permite decir que hay un determinado estado cerebral, al cual designamos rígidamente mediante " $F$ ", no es la observación de $F$ mismo, ni de ninguna propiedad esencial de $F$; dicha evidencia tiene aparentemente sólo una relación contingente con $F$. Es perfectamente imaginable que se diera una situación en la que la evidencia sensorial fuese la misma y sin embargo no se diese F. (Véase 1972, pp. 332-333.)

- Esto no equivale a sostener que la manera como descubrimos en todos los casos que existe un dolor sea porque lo sentimos, pues en el caso de atribuir dolores a terceras personas (si es que éste es un caso de descubrir que existe un dolor) lo hacemos sobre la base de la conducta verbal y no verbal de las terceras personas. No se trata, pues, de una afirmación acerca de cómo sabemos que existe un dolor, es decir, de una afirmación de índole epistemológica, sino de una afirmación con respecto a lo que el dolor es. 
Podemos también imaginar que F fuese capturado mediante evidencias totalmente diferentes. En el caso de identificar un estado cerebral $F$ lo que hacemos es interpretar la evidencia sensorial con la que contamos dentro del marco de una teorfa neurofisiológica. Pero no hay nada que sea identificar a $F$ al margen de una teoría; esto es: no es que existan por ahí, para que se les mire, un conjunto de estados cerebrales determinados y que nuestra única función sea la de seleccionar uno de aquéllos como quien seleccionara, de entre un conjunto de objetos coloreados, aquél que es rojo. En el caso de la identificación del dolor no es necesaria la intervención de ninguna teoría cientifica; en el caso de la identificación del estado cerebral, es indispensable la teoría. De lo anterior parece desprenderse que, si bien la designación del término "dolor" se fija mediante una propiedad esencial de éste, no fijamos la referencia de "estado cerebral F" (estimulación de la fibra $\mathrm{C}$ ) mediante una propiedad esencial de $F$, sino mediante una propiedad contingente de $F$, esto es, por el hecho de estar contingentemente relacionado con una evidencia $\mathrm{E}$. "Ser un estado cerebral de cierto tipo $B$ es $[. .$.$] esencial a B$ (dicho estado cerebral)", dice Kripke, y esto parece inapelable; lo que parece al menos cuestionable es que seleccionemos o identifiquemos dicho estado cerebral por la característica esencial de ser ese estado cerebral, y no por estar correlacionado contingentemente, dentro del marco de una teoría, con determinada evidencia E. ${ }^{10}$

2) En segundo lugar, parece posible que, aun en el caso de las identidades teóricas, fijemos la referencia de sus términos mediante descripciones esenciales y no accidentales. Esto es, dentro de la perspectiva de Kripke, resulta perfectamente posible que la ciencia descubra dos o más propiedades esenciales de un mismo objeto, esto es, propiedades sin las cuales el objeto no sería lo que es. (1972, p. 273; 1971, pp. 151-2 n. 11 y 12.) Podemos decir, por ejemplo, que es esencial al planeta Mercurio tener una composición química $Q$ y que también es esencial a Mercurio estar a una determinada distancia del sol (pues

10 Hay un pasaje en 1972, pp. 338-339, en donde Kripke parece asumir, equivocadamente a mi manera de ver, que en la manera como fijamos la referencia de "la estimulación de las fibras C" interviene el hecho de ser sentida como dolor. Si esto fuese asi y la teoria de la identidad resultase verdadera, efectivamente estaríamos utilizando una propiedad esencial del estado cerebral $F$ ( = dolor) para fijar la designación de "La estimulación de la fibra $C^{\prime \prime}$. Pero esto parece a todas luces falso después de lo argumentado en líneas anteriores: no fijamos la referencia de $\mathrm{F}$ apelando al dolor, $\mathrm{o}$ al hecho de que sea sentida como dolor. En el mismo pasaje, después de haber mostrado Kripke que es posible que el calor hubiese existido sin que se diera la situación que de hecho nos ha permitido fijar la referencia de "calor", esto es, el hecho de que lo experimentamos como caliente, Kripke pregunta: "¿es análogamente posible que la estimulación de la fibra $\mathbf{C}$ pudiera haber existido sin que se hubiese sentido como dolor?" Nótese que la analogía planteada no es correcta; para que lo fuese, la pregunta tendria que haber sido: ¿es análogamente posible que la estimulación de la fibra $C$ pudiera haber existido y que no la seleccionásemos de la manera como de hecho la seleccionamos? La respuesta a esta pregunta es que dicha situación sería obviamente posible. 
de no ser así su constitución química no podrfa ser $Q$ ). Sin embargo, parece francamente contingente la identidad "el planeta cuya constitución química es $\mathbf{Q}=$ el planeta que está a una distancia $d$ del sol". El paradigma de explicación de Kripke no se aplica para disolver la aparente contingencia en estos casos. Aun si aceptásemos que en las identidades psicofísicas las referencias de los términos de la identidad son fijadas mediante propiedades esenciales (cosa que, como vimos, es problemática), tendríamos que, si bien no puede explicarse conforme al modelo anterior la apariencia de contingencia de las identidades psicofísicas, tampoco se explicarla la de algunas identidades teb́ricas como las mencionadas anteriormente.

3) Kripke podría decir que lo que sucede en los casos anteriores es que, asociados a las descripciones "el planeta cuya constitución química es $Q$ " y "el planeta que está a una distancia $d$ del sol", hay ciertos enunciados puramente cualitativos, los cuales son los que permiten explicar la aparente contingencia en estos casos. Estos enunciados puramente cualitativos serían las descripciones de la evidencia sensorial sobre cuya base establecemos la verdad de que Mercurio tiene una constitución qufmica $Q$ y de que Mercurio está a una distancia $d$ del sol (1972, p. 332). Pero si esto es así, también podríamos decir, para el caso de las identidades psicofísicas, que aun cuando ambos designadores que intervienen en ellas fuesen designadores rígidos (por ejemplo, aceptando que fuesen descripciones esenciales), la expresión "estimulación de la fibra C" mediante la cual nos referimos al estado físico (no la descripción mental) está inscrita dentro del marco de una teoría neurofisiológica y asociado, por tanto, a una serie de enunciados que describen también nuestra evidencia sensorial. Ahora bien, la relación entre la descripción teórica - por ejemplo, "la estimulación de la fibra C"- y los enunciados observacionales mediante los cuales fijamos la referencia de dicha descripción, es contingente, pues es perfectamente posible que capturásemos el mismo estado físico mediante otro tipo de observaciones, o, como dice Kripke, es posible que pudiésemos haber estado "cualitativamente en la misma situación epistemológica", esto es, que pudiésemos haber tenido la misma evidencia sensorial, y que sin embargo estuviésemos frente a un tipo de objeto diferente al que de hecho estamos. (1972, p. 332.) ${ }^{11}$ Para mostrar que la contingencia de una identidad teórica es ilusoria, dice Kripke, "nuestro paradigma general es redescribir tanto la evidencia anterior como el enunciado cualitativamente (i. e. en términos de experiencias sensoriales) y sostener que están relacionados sólo contingentemente" (1972, pp. 332-333. Véase también p. 338). Esto sería lo que

11 Héctor-Neri Castañeda sugiere que no se trataria en este caso de identidades estrictas, sino de lo que en su sistema denomina "consustanciaciones". La relación de consustanciación, de acuerdo con Castañeda, es una relación contingente que se descubre a posteriori. Véase su articulo "Thinking and the Structure of the World" en Critica, Revista Hispanoamericana de Filosofia, $n^{\circ} 18,1972$; especialmente pp. 51-57. 
explicaría en último término la impresión de contingencia; pero el problema para Kripke está en que esta explicación disolveria la apariencia de contingecia no sólo de las identidades teóricas sino también la de las psicofísicas.

Si hablamos, por ejemplo, de que el dolor es un patrón 'P determinado de actividad neuronal, no podemos observar dicho patrón, no es algo que esté ahí para que lo miremos, sino que lo que podemos observar son, por. ejemplo, ciertos registros de actividad eléctrica cerebral, o ni siquiera esto, más bien aparatos cuyos indicadores se mueven y agujas que se deslizan sobre un papel para diseñar ciertas gráficas. Ahora bien, es totalmente contingente que esas gráficas estén relacionadas cơn el dolor. Podríamos haber detectado de otras maneras la actividad neuronal. Sostener esto es distinto a sostener que es contingente la relación entre el dolor y P. Kripke señala correctamente que no es posible estar frente a la misma situación epistémica que estamos cuando tenemos un dolor (esto es, la situación en la que sentimos un dolor) y decir sin embargo que no hay dolor; sentir un dolor es suficiente para que exista el dolor. Sin embargo, esto mismo no se puede sostener para el caso del estado cerebral con el que se pretende identificar al dolor; esto es, es posible que estemos frente a una situación epistemológicamente idéntica (cualitativamente descrita) y que en un tiempo $t$ la tomemos como evidencia de la existencia de un estado cerebral designado por " $C$ ", pero que en un tiempo distinto ' $t$ ' se tome como evidencia de otro estado cerebral designado por " $C$ "'. La relación. entre la evidencia y. $\mathrm{C}$ o ' $\mathrm{C}^{\prime}$ sería contingente.

- Las identificaciones teóriças difieren de las psicofísicas (también teóricas) én que laș primeras contienen designadores rigidos de dos fenómenòs externos, en tanto que las segundas pretenden establecer la identidad de una experiencia o tipo de experiencias (subjetivas) con un fénómeno físico o tipo de fenómenos físicos. La impresión de contingencia de esta clase de identidades puede provenir del hecho de que capturamos el fenómeno físico mediante evidencias que pueden estar relacionadas sólo contingentemente con dicho fenómeno, esto es, mediante descripciones accidentales. Si esto es así, puede explicarse entonces la ilusoriedad de la intuición cartesiana diciendo que, si bien sería necesario que $\mathbf{M}=\mathrm{F}$ (en el caso en que las neurociencias llegasen a descubrir el $F$ adecuado), sería contingente que $F$ esté relacionado con la evidencia $E$ (la cual nos permite fijar la referencia de F) y, por tanto, también sería contingente la relación entre $\mathbf{M}$ y $\mathbf{E}$.

Por otro lado, no hay nada formalmente inadecuado en sostener que un mismo objeto $x$ pueda tener necesariamente las propiedades ser $M$ y ser $F$; éstas pueden ser ambas propiedades esenciales de $x$. Si la teoría de la identidad mente-cuerpo ha de considerarse falsa $a$ priori, será porque se muestre que las propiedades ser $\mathrm{M}$ y ser $\mathrm{F}$ son incompatibles, esto es, porque se muestre la imposibilidad de que ( $(\mathrm{Xx})(\mathrm{Mx}$ \& $\mathrm{Fx}$ ). Mientras no se demuestre lo anterior 
podemos seguir sosteniendo, consistentemente, que es posible que exista algo que tenga dos propiedades esenciales, una mental y otra física; por ejemplo, ser un dolor y ser determinado patrón de actividad cerebral.

Margarita M. ValdÉs

INSTITUTO DE INVĖSTICACIONES FILOSÓFICAS

Universidad Nacional Autónoma de México 